\title{
Sinus Lift Augmentation by Using Calcium Sulphate. A Retrospective 12 Months Radiographic Evaluation Over 25 Treated Italian Patients
}

\author{
L. Laino ${ }^{1}$, G. Troiano ${ }^{1}$, G. Giannatempo ${ }^{1}$, U. Graziani ${ }^{1}$, D. Ciavarella ${ }^{1}$, M. Dioguardi ${ }^{1}$, \\ L. Lo Muzio ${ }^{1}$, F. Lauritano ${ }^{2}$ and M. Cicciü ${ }^{2 * *}$ \\ ${ }^{I}$ Department of Clinical and Experimental Medicine, Foggia University, Foggia, Italy \\ ${ }^{2}$ Department of Human Pathology, School of Dentistry, University of Messina, Messina, Italy
}

\begin{abstract}
Purpose: The aim of this investigation was to assess bone healing of sinus lift procedure in which the augmentation has been performed by using calcium sulphate like bone substitutes. The methods of this investigation how the use of Cone Beam Computed Tomography (CBCT) may be a valid instrument to support reconstructive surgery of the jaws. Patients and Methodology: 25 Patients presented large bone defects after tooth extractions located in the upper jaw posterior area. Vertical bone volume was assessed by CBCT examinations before and about six months after sinus lift surgery. Results: Examined defects treated with sinus lift surgery and evaluated by CBCT showed a strong increasing on the bone volume at 6 months follow up control. Conclusion: Calcium sulphate application in sinus lift surgery represent a safe and predictable option in the place of autologous bone. Therefore the application of CBCT investigation may give the clinicians the opportunity of evaluating with high precision value, the consistence of the bone defects before the surgery.
\end{abstract}

Keywords: Autologus bone graft, bone defects, calcium sulphate, cone beam computed tomography, Italian patients, sinus lift.

\section{INTRODUCTION}

The maxillary sinus is a pyramid-shaped cavity occupying the body of the maxilla, laterally surrounded by the nasal wall. The orbital floor is the superior limit while the alveolar process represents the inferior one. Maxillary sinuses drain to the nasal cavity through the "osteum" located in the middle meatus on the medial sinus walls $[1,2]$. The jawbone resorption, consequent to the teeth loose, determines atrophy in height and thickness, by increasing the vertical dimension of occlusion and by reducing the amount of available bone to the implant placement and next prosthesis positioning. The sinus lift surgery by using a lateral window accordingly with Boyne and James technique is still today the most reliable procedure for the bone volume reconstruction of the maxillary atrophic ridge. Sinus augmentation has evolved into a predictable and common surgical modality for increasing the existing height with bone of sufficient quality to allow predictable long term positioning of dental implants. This surgery is a relative safe procedure, but numerous complications may occur as a consequence of improper surgical plan or connected with too aggressive surgical manoeuvres. Several grafting materials have been used over the years like bone substitutes in order to increase the bone volume in atrophic sinus. Even if the Autologous bone have been considered the gold standard in all the bone regenerative techniques, the morbidity caused from the double surgical site has suggested the clinicians to develop other procedures using alloplastic, heterologous materials, and growth factors. [3-7] The goal of this treatment is to obtain sufficient amount

\footnotetext{
*Address correspondence to this author at the Department of Human Pathology, Dental School, Messina University, Via Consolare Valeria 98100, Messina, Italy; Tel: 0039-090 221 6920; E-mail: acromarco@yahoo.it
}

of bone tissue in order to perform a correct implant positioning. Since its first description, numerous papers have been published in this field regarding different grafting materials, modifications to the classic technique, and comparisons between different techniques. Recently, Calcium Sulphate $(\mathrm{CaS})$ has been proposed as bone substitute for being used on regenerative bone procedures. The ratio is related to the fast resorption of the material when used for recovering spacemaking defects. Several published investigation underlined how CaS showed fast replacing with new bone tissue offering better clinical long-term results [5-10].

The aim of this study was to evaluate a radiographic gain after maxillary sinus floor augmentation by lateral approaching, using $\mathrm{CaS}$ as grafting material.

\section{PATIENTS AND METHODOLOGY}

\section{Surgical Procedure}

Patients' exclusion criteria followed the rules of general contraindications to implant surgery like irradiation, chemotherapy or immunosuppressive therapy over the past 5 years, poor oral hygiene and motivation, active periodontitis uncontrolled diabetes, pregnancy or lactation, substance abusers, smoking more than 10 cigarettes per day, psychiatric problems or unrealistic expectations, acute infection in the area intended for implant placement, positive to HIV and hepatitis $\mathrm{B}$ and $\mathrm{C}$, autoimmune diseases such as rheumatoid arthritis, systemic lupus erythematosus, sclerodermia, Sjogren syndrome and dermatomyositis/polymyositis, treated or under treatment with intravenous amino-bisphosphonates, previously subjected to reconstructive procedures of the posterior maxilla and under chronic treatment with steroids or non-steroidal anti-inflammatory drugs signs and symptoms 
of maxillary sinus disease, residual vertical bone. The patients involved in the investigation presented strong pneumatisation of the maxillary sinus, with edentulous ridge vertically resorbed more then $4 \mathrm{~mm}$ or accordingly with class IV of Cawood and Howell [11].

Surgeries were performed at the dental school of the University of Foggia from 2009 to 2013 by two different surgeons. A first cone beam Computed Tomography (CBCT) was performed before sinus lift execution to have a correct preoperative planning. Two weeks before the surgery, all patients underwent oral hygiene instructions and professional hygiene manoeuvres, when necessary. All patients received antibiotics prior to the surgery. Anti-microbial prophylaxis was obtained with the use of 1 gr of Amoxicillin + Clavulanic acid (Augmentin, GlaxoSmithKline, Brentford, Middlesex, United Kingdom) (or erythromycin $500 \mathrm{mg}$ if allergic to penicillin), starting one day before surgery and for the following 4 days. All surgical operations were performed with the aid of an operative microscope. A first crestal full thickness incision and a second mesial vertical one were made through the scalpel number $15 \mathrm{c}$, and a mucoperiosteal flap was elevated. Ostectomy to create the lateral window was performed using a Piezosurgery cutting device (Piezosurgery Medical II; Mectron Medical Technology, Carasco, Italy) with OP3 insert. The detachment of the Schneiderian membrane was initiated starting from the cranial surface and moving in order distally, mesial and palatal finally. Filling of the cavity was performed using calcium sulphate (Surgiplaster, Classimplant ${ }^{\mathbb{B}}$, Rome, Italy). The bone graft material has been applied until the empty cavity results and then a resorbable collagen membrane was placed over. Periosteal incisions were made to release the flaps coronally as needed and were sutured with Vicryl 5.0 sutures until the incisions were perfectly sealed. Patients were advised to follow standard postoperative instructions, including ice-pack placing on the surgical external area, soft high nutrient diet, thorough rinsing with antiseptic mouthwash (chlorhexidine gluconate $0.2 \%$ ). Moreover, they were instructed to avoid sneezing, nose blowing, or other actions that might create high intranasal pressure or vacuum. It was suggested them to avoid drinking with straws for a week. Any kind of prosthesis over the surgical site was not placed for at least a week after surgery to reduce the risk of wound dehiscence. Patients were seen after 10 days for follow-up examinations and sutures removal (Figs. 1-4).

Patients were recalled for additional post- operative checkups 1, 2 and 6 months after the augmentation procedure.

\section{Radiological Evaluation}

All patients performed a radiological examinations ortopanoramic $\mathrm{X}$ ray evaluation (OPT) and a CBCT scan for pre surgical evaluation (Time 0 ). After surgical procedures and a healing period of six months (Time 1) a second CT cone beam scan was performed to assess the vertical bone gain and to plan implant placement. The radiological documentation of each patient was analysed by two different calibrated investigators who performed a linear measurement trough the use of specific software (ONDEMAND3D) to quantify vertical bone gain obtained with the use of $\mathrm{CaS}$ in two stages sinus floor elevation (Fig. 5-8). The software gives the investigators the possibility on evaluating the same area of the treated bone defect by the axial view of the CBCT. The scale of grey has been used to firstly specify the bone defect before the surgery (from the occlusal bone ridge to the Schniderian membrane), and then to evaluate the new height of the same section with the presence of the placed biomaterial.

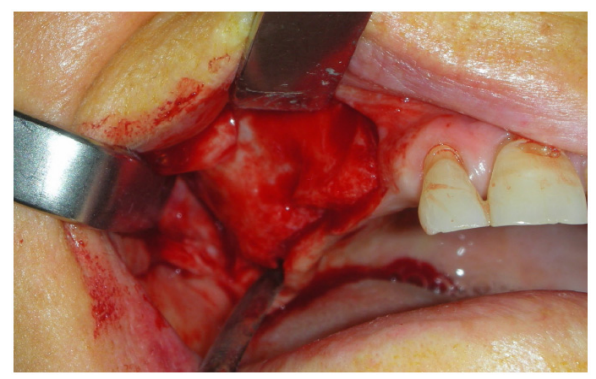

Fig. (1). A full thickness flap is used for exposing the lateral bone before starting the sinus lift surgery.

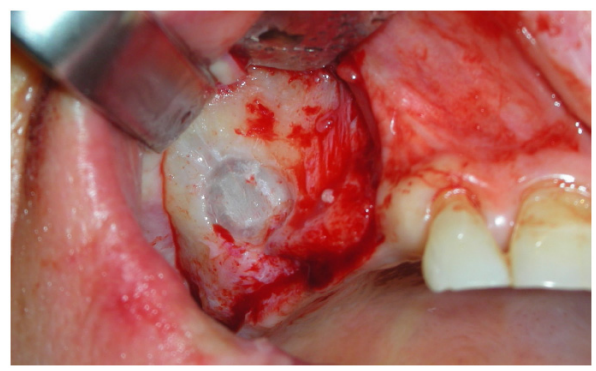

Fig. (2). The osteotomy of the lateral wall shows the design of the window before moving the sinus membrane.

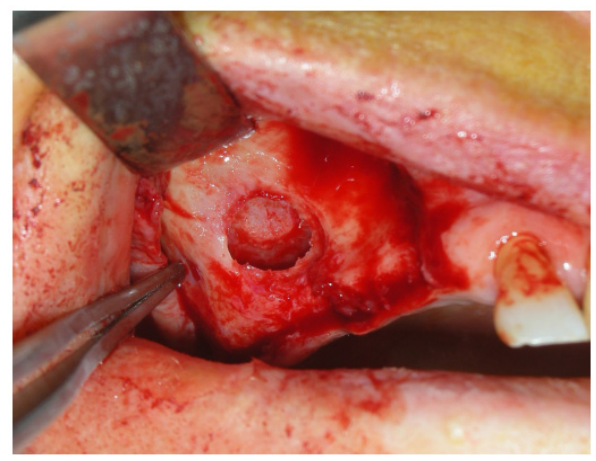

Fig. (3). The membrane is then highlighted and it is possible to fit the material into the created cavity.

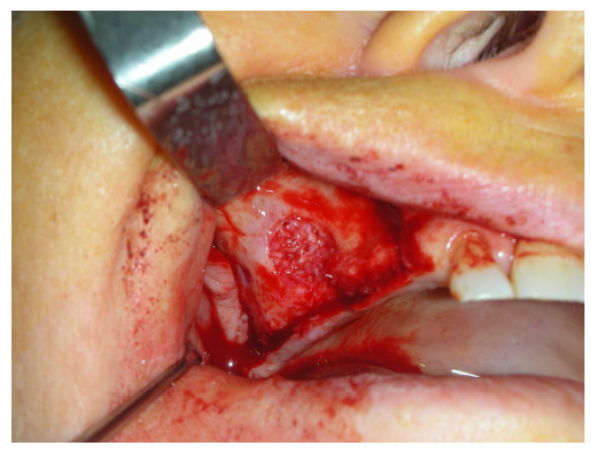

Fig. (4). The bone substitute is then positioned in order to increase the volume of the atrophic maxillary ridge treated. 


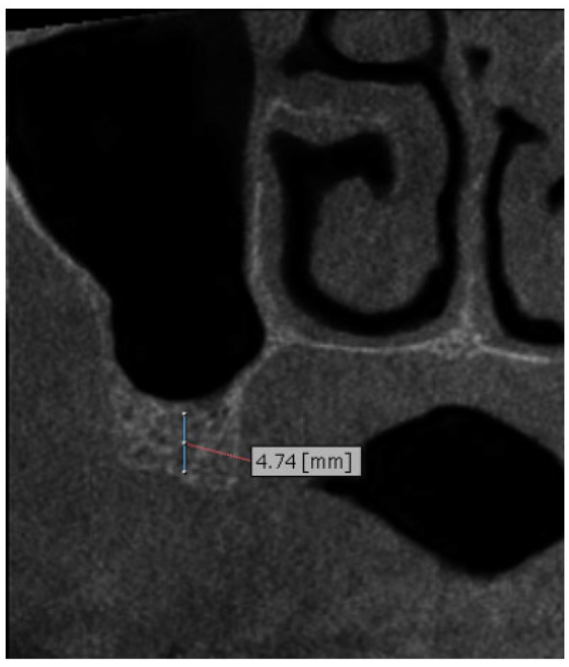

Fig. (5). Sample of preoperative Cone beam radiographic evaluation right side shows the vertical residual ridge like $4.74 \mathrm{~mm}$.

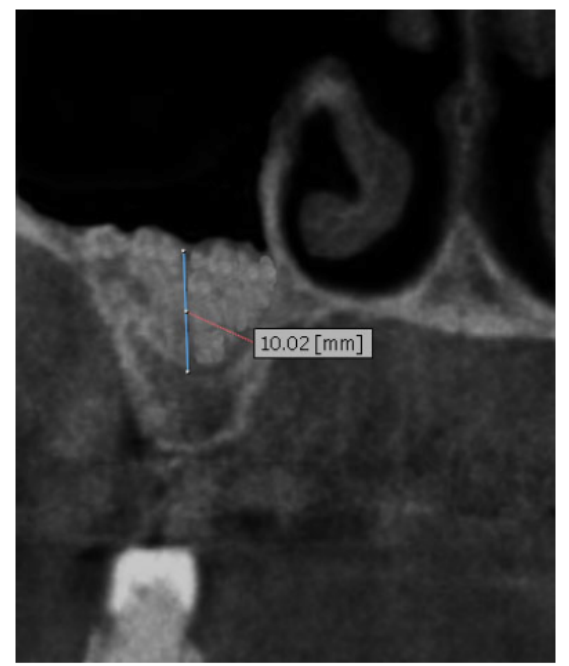

Fig. (6). Sample of 6 months post op Cone beam radiographic evaluation right side shows the vertical residual ridge like 10.02 $\mathrm{mm}$.

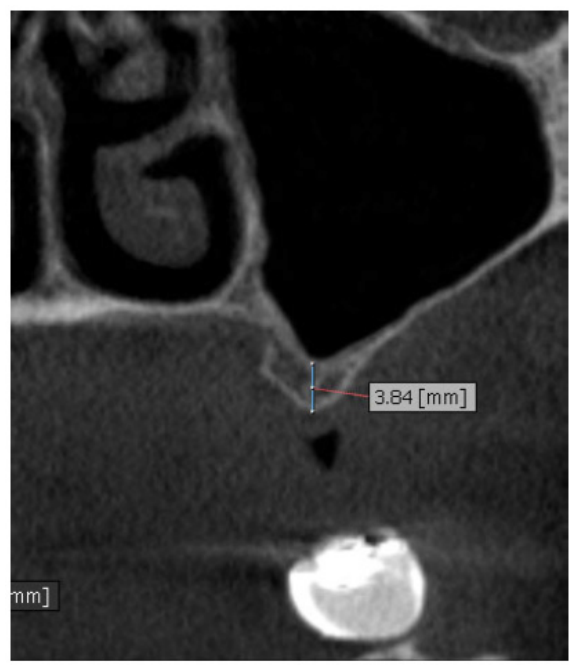

Fig. (7). Sample of preoperative Cone beam radiographic evaluation left sideshows the vertical residual ridge like $3.84 \mathrm{~mm}$.

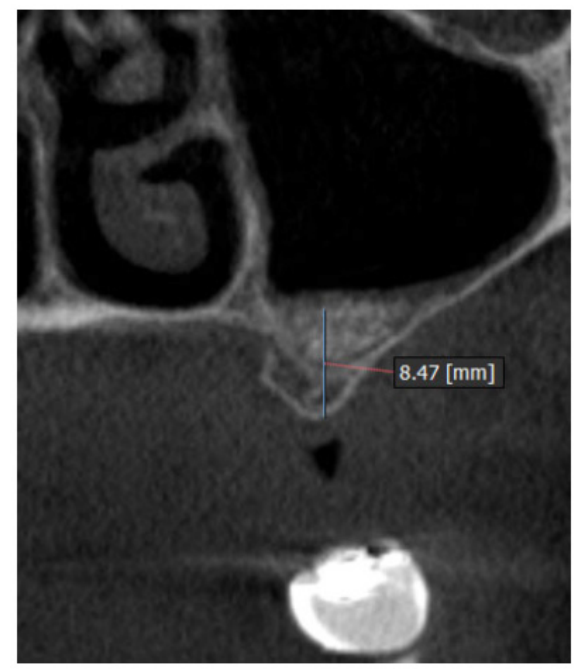

Fig. (8). Sample of 6 months post op Cone beam radiographic evaluation left sideshows the vertical residual ridge like $8.47 \mathrm{~mm}$.

20 pre and post surgery radiographic sections were recorded for each patient. All the data were presented as mean +/- standard deviations and examined with use of Microsoft Excel software.

\section{RESULTS}

Twenty-seven patients with a mean age of 59 years (range 49-75) were considered eligible and enrolled in the study. Two patients were excluded from the study because they refused to perform a secondary CT cone beam scan. One patient present a intra operative complication with membrane perforation resolved trough positioning of resorbable membrane BioGide ${ }^{\circledR}$ (Geistlish Pharmaceutical, Wolhusen, Switzerland) in order to complete the augmentation procedure. No other complications were observed during and after the surgical procedures. For each patient it was measured pre and post surgical vertical bone height in order to quantified the mean and standard deviation of bone gain. The same operator performed all the measurements at Time 0 and Time 1 accordingly with the previous presented protocol. Data were summarized in the Table 1. The mean vertical residual bone before surgery was $4,04 \pm 1,48 \mathrm{~mm}$ while the mean of regenerated sites was $12,25 \pm 3,20 \mathrm{~mm}$, so the mean of bone gain was $8,21 \pm 1,73 \mathrm{~mm}$.

Table 1. Explanations of the patients recorded for the study. The mean of the pre surgery height of the bone ridge is compared with the post op one, in order to have the mean height obtained after all the surgeries.

\begin{tabular}{|c|c|c|c|}
\hline $\mathbf{N}^{\circ}$ Patients & $\begin{array}{c}\text { Mean presurgi- } \\
\text { cal height }\end{array}$ & $\begin{array}{c}\text { Mean postsurgi- } \\
\text { cal height }\end{array}$ & Mean bone gain \\
\hline \hline 25 & $4,04 \pm 1,48 \mathrm{~mm}$ & $12,25 \pm 3,20 \mathrm{~mm}$ & $8,21 \pm 1,73 \mathrm{~mm}$ \\
\hline
\end{tabular}

Data are presented as mean $+/$ - standard deviations.

\section{DISCUSSION}

The results of the study may give important information about the biomaterial used in the surgery but at the same 


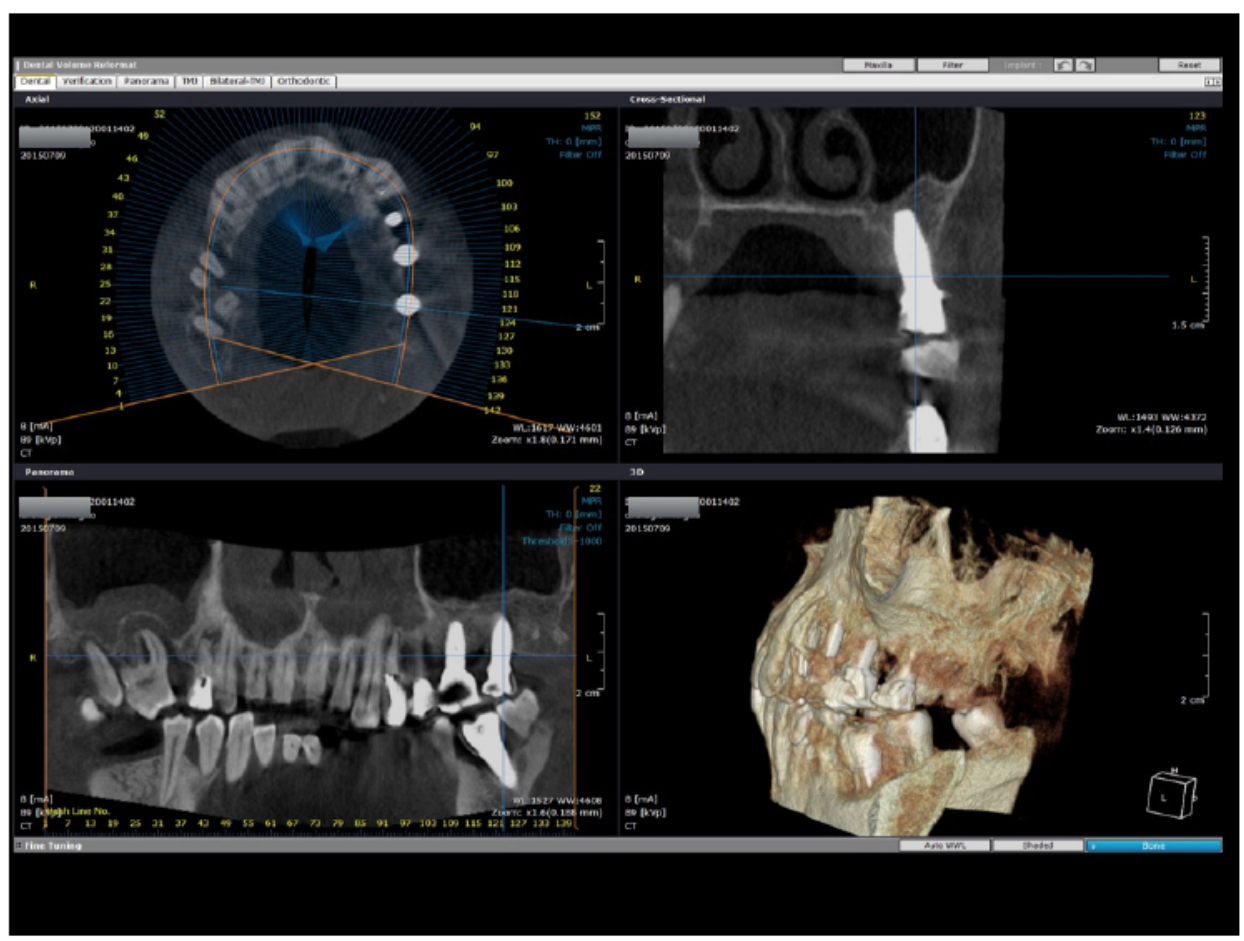

Fig. (9). 1 year post op evaluation. The two dental implants are well integrated in the newly formed bone. A particular of the implant placed in the distal position.

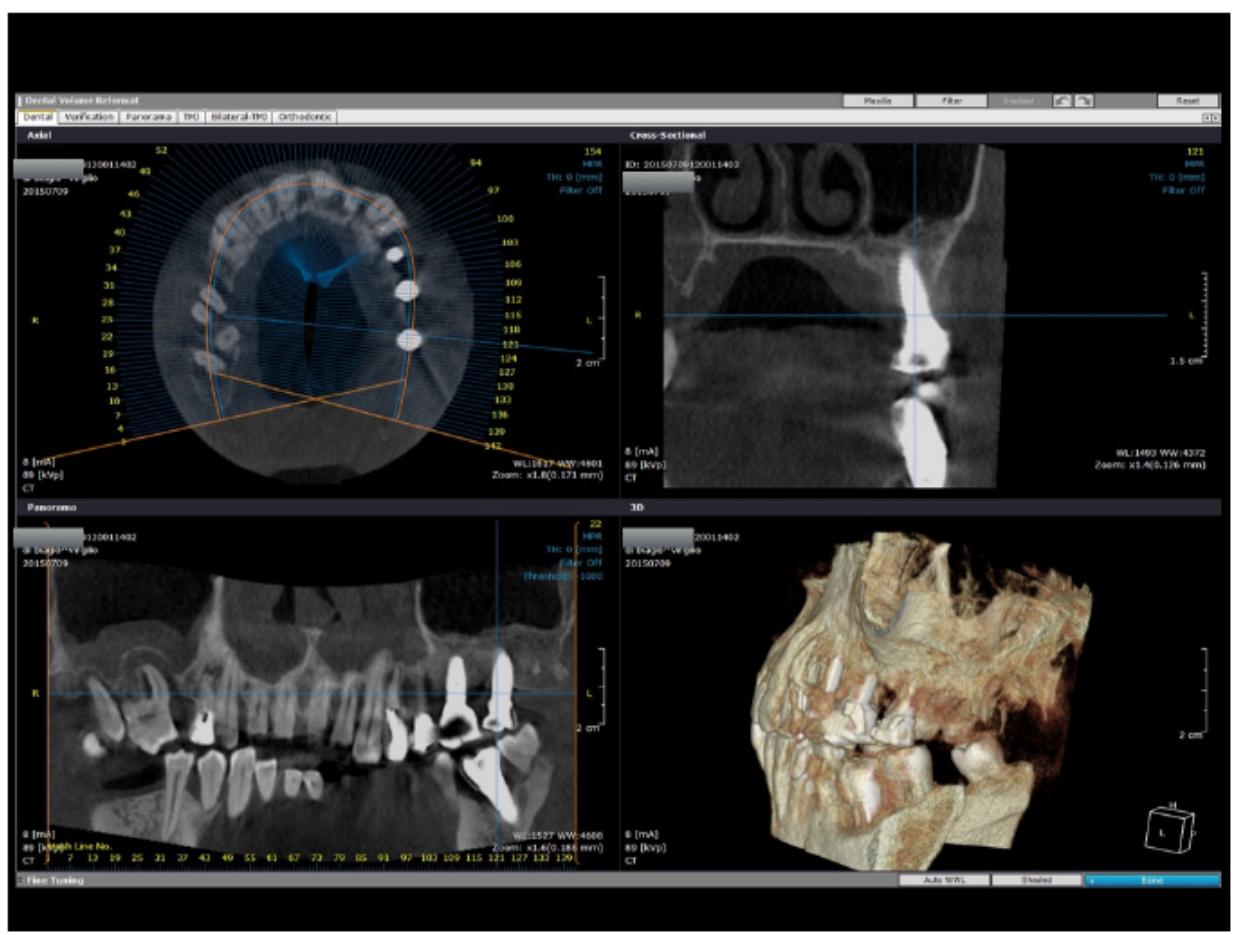

Fig. (10). 1 year post op evaluation. The two dental implants are well integrated in the newly formed bone. A particular of the implant placed in the mesial position.

time the study presents several limitation. The healing time is 6 months and it is ideal for placing the dental implant but at the same time, next radiological follow up after 12, 24, 36 months will be performed and recorded in order to evaluate the stability of the bone graft in the time. The Figs. $(\mathbf{9}, \mathbf{1 0})$ clearly underlined how the newly formed bone in the aug- mented sinus gives high stability even after the prosthodontics restoration of the two dental implants placed. Ideal bone substitute should have the following characteristics: it should be biocompatible and bioresorbable; it should have osteoconductive or osteoinductive properties; and it should be replaced by newly formed bone. Many type of biomaterials 
have been used during the last decades. However the gold standard it still considered the autologous bone for its osteoconductive, osteogenetic and osteoinductive properties [12]. Nevertheless, autogenous bone needs to be taken from an intra or extra oral donor site [13]. This required a second surgical act, resulting in a pro- longed procedural time and greater human resources when compared to grafting with other bone substitutes. In literature have been described, permanent and temporary sensory injuries in the region of the bone levy $[14,15]$. Nowadays the use of piezosurgery device cause less discomfort for the patients compared to traditional techniques but determine increased surgical time $[16,17]$. To avoid this clinical complication a large number of biomaterials have been used as filler in sinus lift augmentation. For its properties of biocompatibility and resorption, associated with a relative low cost, Calcium Sulphate is consider a safe and predictable bone substitute [18]. The clinical use of Calcium sulphate has a longer history than most of the currently available biomaterials. The first internal use to fill bony defects was reported in 1892 by Dreessmann [19]. In literature this material has been used for a large kind of surgical procedures like: correction of periimplant bone defects [20], periodontal tissue regeneration [18], and as substitute in a sinus lift augmentation [21]. Guarnieri et al. in 2006 reports the radiographic and histologic data of 10 patients treated with granular calcium sulfate as a grafting material for maxillary sinus augmentation [22]. In this study, authors described a complete resorption of the biomaterial 6 months after surgery. Between 5 and 6 months postsurgery, the grafted material became radiographically invisible and the grafted area showed a new irregular trabecular design. Iezzi et al. 2007 histologically evaluated an immediately loaded provisional implant retrieved six months after simultaneous placement in a human sinus augmented with CaS. They found a native and newly formed bone with small osteocytes lacunae in contact with the implant surfaces using low-power magnification [22].

The aim of the present study was to evaluate a tridimensional radiographic gain after biphasic maxillary sinus floor augmentation, using $\mathrm{CaS}$ as grafting material. It was observed that the mean vertical residual bone before surgery was 4,04 $\pm 1,48 \mathrm{~mm}$ while the mean of regenerated sites was $12,25 \pm 3,20 \mathrm{~mm}$, so the mean of bone gain was $8,21 \pm 1,73$ $\mathrm{mm}$. These results suggest that the use of $\mathrm{CaS}$ as bone filler in sinus lift augmentation determines a good improve of amount of available bone. In fact, in all cases it was possible to realize a correct implants positioning. Indeed, in this study we don't observe any kind of clinical and radiological complications related to the use of $\mathrm{CaS}$ during the 6 months healing period.

\section{CONCLUSION}

There are different surgical methods for performing the sinus augmentation; the factors that contribute to the survival rate of sinus augmentation and dental implant placement are still the subject of discussion. The success may be related to several parameters like anatomical, surgical or procedural causes. The bone graft material seems to play a role in the next long term success of the surgical procedure. The recent literature concerning sinus grafts has shown differing longterm results depending on which type of bone-graft material was used. [10-12] An ideal maxillary sinus bone-grafting material should provide biologic stability, ensure volume maintenance, and allow the occurrence of new bone infiltration and bone remodelling. Over time, bone-grafting materials and implants should achieve osseointegration. After the restoration of the upper part of the implant has been completed, there should be no bone loss and the materials should be stable; there should be a predictable success rate. In this clinical trial, it was recorded a mean improve of $8,21 \mathrm{~mm}$ in the regenerated sites at six months post op control after the regenerative procedure. The present study suggest that the use of $\mathrm{CaS}$ in biphasic sinus augmentation procedures obtain an unfading and durable improve of newly bone formation.

\section{CONFLICT OF INTEREST}

The authors confirm that this article content has no conflict of interest.

\section{ACKNOWLEDGEMENTS}

Declared none.

\section{REFERENCES}

[1] Guncu GN, Yildirim YD, Wang HL, Tozum TF. Location of posterior superior alveolar artery and evaluation of maxillary sinus anatomy with computerized tomography: a clinical study. Clin Oral Implants Res 2011; 22: 1164-7.

[2] Park YB, Jeon HS, Shim JS, Lee KW, Moon HS. Analysis of the anatomy of the maxillary sinus septum using 3-dimensional computed tomography. J Oral Maxillofac Surg 2011; 69: 1070-8.

[3] Farina R, Pramstraller M, Franceschetti G, Pramstraller C, Trombelli L. Alveolar ridge dimensions in maxillary posterior sextants: a retrospective comparative study of dentate and edentulous sites using computerized tomography data. Clin Oral Implants Res 2011; 22: 1138-44.

[4] Tolman DE. Advanced residual ridge resorption: surgical management. Int J Prosthodont 1993; 6: 118-25.

[5] Esposito M, Grusovin MG, Rees J, et al. Interventions for replacing missing teeth: augmentation procedures of the maxillary sinus. Cochrane Database Syst Rev 2010; 17(3): CD008397.

[6] Shanbhag S, Shanbhag V, Stavropoulos A. Volume changes of maxillary sinus augmentations over time: a systematic review. Int $\mathbf{J}$ Oral Maxillofac Implants 2014; 29: 881-92.

[7] Boyne PJ, James RA. Grafting of the maxillary sinus floor with autogenous marrow and bone. J Oral Surg 1980; 38: 613-6.

[8] Merli M, Moscatelli M, Mariotti G, Rotundo R, Nieri M. Autogenous bone versus deproteinised bovine bone matrix in 1stage lateral sinus floor elevation in the severely atrophied maxilla: a randomised controlled trial. Eur J Oral Implantol 2013; 6: 27-37.

[9] Almaiman M, Al-Bargi HH, Manson P. Complication of anterior iliac bone graft harvesting in 372 adult patients from may 2006 to may 2011 and a literature review. Craniomaxillofac Trauma Reconstr 2013; 6: 257-66.

[10] Esposito M, Felice P, Worthington HV. Interventions for replacing missing teeth: augmentation procedures of the maxillary sinus. Cochrane Database Syst Rev 2014; 5: CD008397.

[11] Cawood JI, Howell RA. A classification of the edentulous jaws. Int J Oral Maxillofac Surg 1988; 17(4): 232-6.

[12] Berberi A, Samarani A, Nader N, et al. Physicochemical characteristics of bone substitutes used in oral surgery in comparison to autogenous bone. Biomed Res Int 2014; 2014: 320790 .

[13] Peng W, Kim IK, Cho HY, et al. Assessment of the autogenous bone graft for sinus elevation. J Korean Assoc Oral Maxillofac Surg 2013; 39: 274-82.

[14] Fakhry A. The mandibular retromolar area as a donor site in maxillofacial bone grafting: surgical notes. Int $\mathrm{J}$ Periodontics Restorative Dent 2011; 31: 275-83. 
[15] Beretta M, Cicciù M, Bramanti E, Maiorana C. Schneider membrane elevation in presence of sinus septa: anatomic features and surgical management. Int J Dent 2012; 2012: 261905.

[16] Pereira CC, Gealh WC, Nogueira LM, Garcia Junior IR, Okamoto R. Piezosurgey applied to implant dentistry: clinical and biological aspects. J Oral Implantol 2014; 40: S1 401-8.

[17] Majewski P. Piezoelectric surgery in autogenous bone block grafts. Int J Periodontics Restorative Dent 2014; 34: 355-63.

[18] Orsini M, Orsini G, Benlloch D, et al. Comparison of calcium sulfate and autogenous bone graft to bioabsorbable membranes plus autogenous bone graft in the treatment of intrabony periodontal defects: a split-mouth study. J Periodontol 2001; 72: 296-302.
[19] Kutkut A, Andreana S. Medical-grade calcium sulfate hemihydrate in clinical implant dentistry: a review. J Long Term Eff Med Implants 2010; 20: 295-301.

[20] Scarano A, Orsini G, Pecora G, Iezzi G, Perrotti V, Piattelli A. Peri-implant bone regeneration with calcium sulfate: a light and transmission electron microscopy case report. Implants Dent 2007; 16: 195-203.

[21] Maridati P, Stoffella E, Speroni S, Cicciu M, Maiorana C. Alveolar antral artery isolation during sinus lift procedure with the double window technique. Open Dent J 2014; 8: 95-103.

[22] Guarnieri R, Grassi R, Ripari M, Pecora G. Maxillary sinus augmentation using granular calcium sulfate (surgiplaster sinus): radiographic and histologic study at 2 years. Int $\mathrm{J}$ Periodontics Restorative Dent 2006; 26: 79-85.

Received: June 12, 2015

(C) Laino et al.; Licensee Bentham Open.

This is an open access article licensed under the terms of the (https://creativecommons.org/licenses/by/4.0/legalcode), which permits unrestricted, noncommercial use, distribution and reproduction in any medium, provided the work is properly cited. 